\title{
Diffuse large-scale extragalactic radio structures
}

\section{Marek Jamrozy*, Marek Weżgowiec}

Jagiellonian University, ul. Orla 171, PL-30244 Kraków, Poland

E-mail: jamrozy@oa.uj.edu.pl, markmet@oa.uj.edu.pl

\section{Karl-Heinz Mack}

INAF - Istituto di Radioastronomia, Via P. Gobetti 101, I-40129 Bologna, Italy

E-mail: mackeira.inaf.it

Galaxies with active galactic nuclei are usually powerful synchrotron emitters. A small fraction of radio galaxies with linear sizes of more than $1 \mathrm{Mpc}$ represent the biggest single objects in the Universe. In addition, at least some of these giants are surrounded by weak diffuse radio cocoons. By observing them we may acquire important information about the formation and evolution of radio galaxies (RGs) as well as about the composition of the inter-galactic medium (IGM) on scales comparable to those of clusters of galaxies or larger. Unfortunately, steep radio spectra and low surface-brightness of such sources make them difficult to observe. It will therefore be important to observe such objects with the Square Kilometer Array (SKA). With its extraordinary sensitivity it will provide us with valuable data, especially at lower frequencies of 100 - 500 MHz. In this paper we show examples of diffuse radio halos around some extended Giant Radio Galaxies (GRGs).

From Planets to Dark Energy: the Modern Radio Universe October 1-5 2007

The University of Manchester, $U K$

* Speaker. 


\section{Introduction}

There is a huge range from less than $100 \mathrm{pc}$ up to a few Mpc in linear extent of RGs. RGs with sizes over $1 \mathrm{Mpc}$ are named GRGs. The most extreme of those is 3C 236 which has a projected linear size of $4.2 \mathrm{Mpc}\left(\mathrm{H}_{0}=71 \mathrm{~km} \mathrm{~s}^{-1} \mathrm{Mpc}^{-1}, \Omega=1\right)$. The very large angular sizes (up to some dozens of arc minutes) of several GRGs on the sky give an excellent opportunity to study the nature of active galactic nuclei (AGNs) and provide important constraints on the evolution of galaxies. Although significant progress has been made in our understanding of the evolution of radio sources, it is not yet clear which conditions lead to the formation of a GRG.

\section{Extended radio cocoons and observational problems with the present day radio telescopes}

Recent observations suggest that around at least some GRGs extended diffuse cocoons may occur. They may provide important clues about the structure and the evolution of these sources. Neglecting the presence of those cocoons can cause serious under- or overestimations of various physical parameters of RGs such as their entire volumes, total luminosities, radiative ages, etc. Since such halos are thought to have low surface brightness, steep synchrotron spectra and large extent on the sky they are hidden from present day investigations due to existing observational problems. Present instruments do not provide sufficient dynamic range to allow detection of faint diffuse structure and strongly emitting features at the same time. Moreover, interferometer observations of extended RGs bring poor sensitivity for extended structures due to the so-called "missing zero spacing" effect (see Figs. 1a and 2a). The larger the extent of the observed source the bigger both structure and flux-density losses. For source sizes of over 15 arc minutes (typical extent for low-redshift GRGs) even one third of the total flux density can be lost. This situation can naturally be avoided in using single-dish telescopes which are sensitive to extended structures. However, even the largest $(\sim 100 \mathrm{~m})$ instruments provide very low angular resolution (of the order of one to tens of arc minutes). Therefore, it is hard to get detailed information about the real morphology of particular sources based on single-dish maps. To some extent, we could improve our observations by combining interferometric and single-dish data made at the same frequency. This treatment allows us to produce high-resolution maps with satisfying sensitivity for extended structures (see Fig. $1 \mathrm{~b}$ and 2b). Nevertheless, only advanced instruments can meet all the current needs of investigations of GRGs.

\section{Future perspectives for GRGs investigations}

The small number of well-studied GRGs so far is a reason for a gap in our understanding of RG evolution, hence the phenomenon of GRGs still awaits its comprehensive explanation. Because GRGs typically are low surface-brightness objects, until now only few large angular-size GRGs with extended halos have been mapped at low radio frequencies. It is important to study these sources at frequencies of $100-500 \mathrm{MHz}$ as well as in the $\mathrm{GHz}$ regime with high sensitivity and good angular resolution. The proposed SKA telescope will be an excellent tool for investigating GRGs and their diffuse cocoons. 

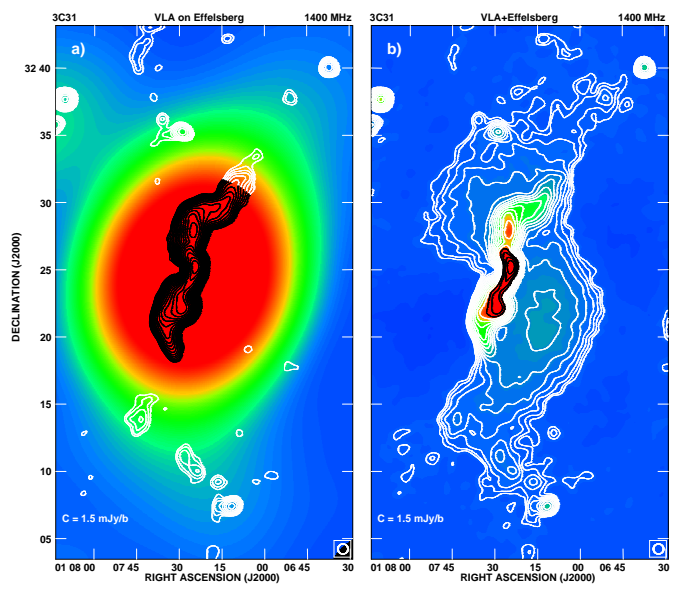

Figure 1: 3C 31 at $1400 \mathrm{MHz}$. a: NVSS contours [1] overlaid onto the Effelsberg color map. This picture shows the insensitivity of interferometers to weak extended emission of the RG as well as the poor resolution of single-dish observations. b: Combined NVSS and Effelsberg data (both contours and colors). Some of the extended Effelsberg structures have been re-added to the high-resolution NVSS map.
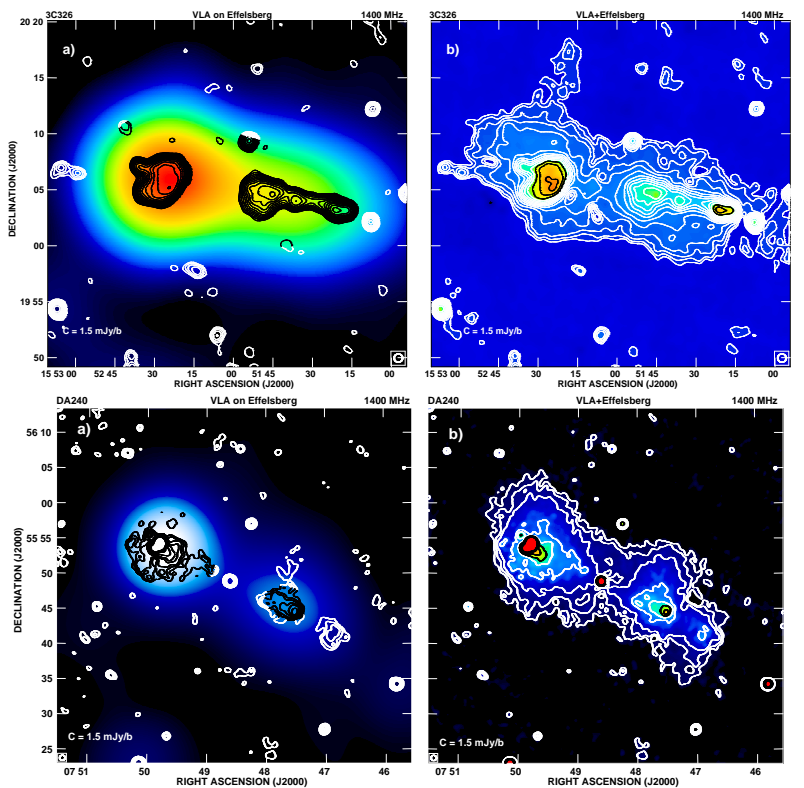

Figure 2: Similar as in Fig. 1 but upper panel: for the GRG 3C 326 and lower panel: for the GRG DA 240.

\section{References}

[1] J.J. Condon, W.D. Cotton, E.W Greisen, Q.F. Yin, R.A. Perley, G.B. Taylor, J.J. Broderick, AJ 115 (1998) 1693. 